\title{
Distinct patterns of diversity, population structure and evolution in the AMA1 genes of sympatric Plasmodium falciparum and Plasmodium vivax populations of Papua New Guinea from an area of similarly high transmission
}

Alicia Arnott ${ }^{1,11,12}$, Johanna Wapling ${ }^{1}$, Ivo Mueller ${ }^{2,3,4}$, Paul A Ramsland ${ }^{1,5,6,7}$, Peter M Siba ${ }^{8}$, John C Reeder 9,10 and Alyssa E Barry $3,4^{*}$

\begin{abstract}
Background: As Plasmodium falciparum and Plasmodium vivax co-exist in most malaria-endemic regions outside sub-Saharan Africa, malaria control strategies in these areas must target both species in order to succeed. Population genetic analyses can predict the effectiveness of interventions including vaccines, by providing insight into patterns of diversity and evolution. The aim of this study was to investigate the population genetics of leading malaria vaccine candidate AMA 1 in sympatric $P$. falciparum and $P$. vivax populations of Papua New Guinea (PNG), an area of similarly high prevalence ( $P f=22.3$ to $38.8 \%, P V=15.3$ to $31.8 \%$ ).
\end{abstract}

Methods: A total of 72 Pfama1 and 102 Pvamal sequences were collected from two distinct areas, Madang and Wosera, on the highly endemic PNG north coast.

Results: Despite a greater number of polymorphic sites in the AMA1 genes of $P$. falciparum (Madang $=52$; Wosera $=56$ ) compared to $P$. vivax (Madang $=36$, Wosera $=34$ ), the number of AMA1 haplotypes, haplotype diversity $(H d)$ and recombination $(R)$ was far lower for $P$. falciparum (Madang $=12$, Wosera $=20 ; H d \leq 0.92, R \leq 45.8$ ) than for $P$. vivax (Madang $=50$, Wosera $=38 ; H d=0.99, R=\leq 70.9$ ). Balancing selection was detected only within domain I of AMA1 for $P$. vivax, and in both domains I and III for P. falciparum.

Conclusions: Higher diversity in the genes encoding P. vivax AMA1 than in P. falciparum AMA1 in this highly endemic area has important implications for development of AMA1-based vaccines in PNG and beyond. These results also suggest a smaller effective population size of $P$. falciparum compared to $P$. vivax, a finding that warrants further investigation. Differing patterns of selection on the AMA1 genes indicate that critical antigenic sites may differ between the species, highlighting the need for independent investigations of these two leading vaccine candidates.

Keywords: Plasmodium falciparum, Plasmodium vivax, AMA1, Malaria vaccine, Population genetics, Papua New Guinea

\footnotetext{
* Correspondence: barry@wehi.edu.au

${ }^{3}$ Division of Infection and Immunity, Walter and Eliza Hall Institute of Medical Research, Melbourne, Australia

${ }^{4}$ Department of Medical Biology, University of Melbourne, Parkville, Australia

Full list of author information is available at the end of the article
}

\section{Biomed Central}

(c) 2014 Arnott et al.; licensee BioMed Central Ltd. This is an Open Access article distributed under the terms of the Creative Commons Attribution License (http://creativecommons.org/licenses/by/4.0), which permits unrestricted use, distribution, and reproduction in any medium, provided the original work is properly credited. The Creative Commons Public Domain Dedication waiver (http://creativecommons.org/publicdomain/zero/1.0/) applies to the data made available in this article, unless otherwise stated. 


\section{Background}

Although greater morbidity and mortality from malaria is attributed to infection with Plasmodium falciparum, Plasmodium vivax is responsible for much of the global malaria burden. This species can also cause severe disease [1-4], has a broader geographic distribution than $P$. falciparum and is increasingly being recognized as less responsive to malaria control measures [5]. Indeed, in the context of malaria elimination, it is thought that the relatively resilient $P$. vivax will present the ultimate challenge in areas in areas of co-endemicity [6].

The greater resilience of $P$. vivax is thought to be predominantly due to its ability to form dormant liver stages [7] and may also be because P. vivax is a more genetically diverse parasite than $P$. falciparum. The greater diversity of $P$. vivax has been demonstrated using a panel of global isolates [8] and in an endemic area of Cambodia with similarly low transmission of both species $[9,10]$. Diversity is an indicator of the evolutionary fitness of a parasite population as high genetic diversity provides greater potential for adaptation to changing environmental conditions and for immune escape, which is facilitated by antigenic polymorphism [11]. Plasmodium falciparum diversity is strongly linked with the overall transmission intensity of the broad geographic area [12] and is consistent with decreasing diversity with distance from an African origin [13]. However, diverse P. vivax populations have been observed even in very low transmission settings $[14,15]$ and the origin of $P$. vivax is still being debated [16-18]. Defining the genetic diversity of $P$. falciparum and $P$. vivax parasite populations can therefore provide important insight into malaria epidemiology and parasite evolution, in addition to how well the local parasite population might respond to interventions such as malaria vaccines [19].

A broadly effective malaria vaccine is considered the most sustainable approach to controlling and eventually eliminating malaria [20]. One of the major barriers to the development of malaria vaccines is the extreme diversity of leading candidate antigens. Therefore, a better understanding of parasite antigenic diversity is urgently needed $[21,22]$. As $P$. falciparum and $P$. vivax co-exist in many malaria-endemic regions, it is essential that both species be targeted if elimination is to be achieved. Whilst many leading malaria vaccine candidates are orthologous, on account of biological differences that exist between the species it is not possible to extrapolate findings from one species to the other [8,23-25]. Therefore, the antigenic diversity of leading candidates must be investigated independently for each species.

One of the most promising vaccine candidates for both $P$. falciparum and $P$. vivax is the apical membrane antigen 1 (AMA1) [26-33]. Although important for merozoite invasion, the precise biological function of AMA1 was largely unknown until it was recently reported that
P. falciparum AMA1 (PfAMA1) is essential for invasion of host cells as it has a direct or indirect role in resealing of the red blood cell at the posterior end of the invasion event [34]. That AMA1 sequences are highly conserved amongst all parasites of the Apicomplexa phylum suggests conservation of fundamental biological properties [35]. Additionally, both the P. falciparum [36-38] and $P$. vivax [39-42] AMA1 ectodomains are highly immunogenic. PfAMA1 elicits antibodies that can inhibit invasion of host cells in vitro [43-45], thereby contributing to protective immunity in humans naturally exposed to malaria $[46,47]$. AMA1 is, therefore, considered to be a prime vaccine candidate for both $P$. falciparum and $P$. vivax, however few studies have investigated the genetic diversity of AMA1 in sympatric $P$. falciparum and $P$. vivax populations $[48,49]$. In Venezuela, the genes encoding $P$. vivax AMA1 (PvAMA1) were reported to be significantly more diverse than PfAMA1. However, $P$. vivax has a much higher prevalence than $P$. falciparum in this region, which may explain the patterns observed [48]. The diversity of each species at the ama1 locus is yet to be compared in an area highly endemic for both species.

Intense year-round transmission of both $P$. falciparum and $P$. vivax occurs on the north coast of Papua New Guinea (PNG) [50]. Separate population genetic studies of AMA1 have been done in this area for $P$. falciparum $[21,35]$ and $P$. vivax $[51,52]$ however the results cannot be directly compared on account of samples being collected at different time points and because only partial ama1 sequences were analysed for P. falciparum. The findings of these previous studies revealed that PNG parasites have a genetically distinct repertoire of $P$. vivax AMA1 alleles compared to other populations [51], whereas P. falciparum AMA1 domain I (DI) sequences collected in the year 2000 from a single, highly diverse parasite population [35] were found to be representative of the worldwide diversity [21].

The aim of this study was to investigate patterns of diversity, population structure and evolution of full-length $P$. falciparum and P. vivax AMA1 genes in sympatric parasite populations in two geographically distinct areas of PNG. Analysis of the data confirmed and extended previous findings, revealing important differences in the population biology of $P$. falciparum and $P$. vivax in PNG which may have important implications for the design of AMA1-based vaccines.

\section{Methods}

\section{Study sites and isolates}

The Madang and East Sepik Provinces on the north coast of PNG are areas of intense perennial malaria transmission and have been the focus of malaria research and control efforts for more than half a century. Cross-sectional malaria surveys including asymptomatic volunteers of all ages were conducted in 2005 and 2006 
in four catchment areas including Mugil, Malala and Utu in the Madang Province, and the Wosera district in the East Sepik Province. The study sites and parasite isolates have been described in detail elsewhere [53-55]. Plasmodium falciparum- and P. vivax-infected samples were identified by PCR-based methods [54,55]. The prevalence of both species was found to be similarly high at 22.3 to $38.8 \%$ for P. falciparum [55] and 15.3 to $31.8 \%$ for $P$. vivax [54]. Isolates were also genotyped at highly polymorphic loci to identify monoclonal infections as previously reported [53,54]. A total of 76 monoclonal P. falciparum [53] and 102 monoclonal P. vivax isolates [54] were selected for analysis in this study. Using microsatellite markers, a moderate to high degree of population structure for $P$. falciparum (i.e. high diversity between populations [53]) but limited geographic population structure of $P$. vivax was identified in this region (i.e. limited diversity between populations [56]). Therefore, for $P$. falciparum only isolates from Mugil were used, and due to the relatively small number of $P$. vivax samples available from each of the three Madang catchments, isolates were combined to form a single population for analysis. Catchment populations were also analysed separately to confirm the patterns observed. The two distinct parasite populations will be referred to as Wosera and Madang throughout the manuscript.

Ethical approval to conduct this study was granted by the PNG Institute of Medical Research Institutional Review Board (Nos 08-08 and 11-05), the Medical Research Advisory Committee of PNG (Nos 10.23 and 11-06), the Alfred Hospital Research and Ethics Unit (No 30/06Q and 420-10) and the Walter and Eliza Hall Institute Human Research Ethics Committee (No 11-09 and 11-12).

\section{PCR and sequencing}

Whole genome amplification (WGA) was performed for all $P$. falciparum samples using the Illustra GenomiPhi V2 Amplification kit (GE Healthcare, NSW, Australia), as per the manufacturer's instructions. The multiple displacement amplification (MDA) WGA method used has been shown to result in a higher yield of non-artifact DNA templates and reduced amplification bias compared with PCR-based WGA methods [57]. Previously, to investigate whether artifacts were introduced as a result of WGA, $P$. vivax sequences obtained from undiluted genomic DNA and sequences amplified from WGA template were compared [54]. Consistent with previous reports [58,59], results were concordant between the WGA and unamplified DNA. Nucleotides 38 to $1,674 \mathrm{bp}$ of the $1,869 \mathrm{bp}$ Pfama1 coding sequence, encompassing the prosequence, signal sequence and the complete ectodomain (DI to DIII) were amplified using a modified version of a previously described nested PCR strategy $[60,61]$. Modifications included: both primary
PCR primers Fex (5'-ATGTACTTGTTATAAATTGTAC-3' (Fwd)) and Rex (5'-CAGCTTCTCTTTTATGCTAA-3' $(\operatorname{Rev})$ ). For the nested PCR, the published forward primer F2 [60] was used with the TMr reverse primer (5'-GC TGTCGCTGTATTAGCAACTA-3'). As the $P$. falciparum genome is extremely AT-rich, if PCR was unsuccessful, the PCR enhancers, dimethyl sulphoxide (DMSO, 5\% PCR grade; Sigma-Aldrich, MO, USA), Betaine (0.05 M PCR grade; Sigma-Aldrich, MO, USA), bovine serum albumin (BSA, $0.1 \mathrm{mg} / \mathrm{mL}$ PCR grade; Roche, $\mathrm{GmbH}$, Germany) or polyethylene glycol (PEG6000, 1.5\%; Sigma-Aldrich, MO, USA) were added to the PCR mastermix. Sequencing reactions were performed by a contract sequencing facility using the ABI BigDye Terminator Cycle Sequencing kit on an ABI 3730XL automatic DNA Analyser (Macrogen, Seoul, Korea). For $P$. vivax, nucleotides 1 to 1,524 bp of the 1,686 bp Pvama1 coding sequence, encompassing the signal sequence and the complete ectodomain (DI to DIII) were amplified and sequenced previously as described $[48,51,62]$.

\section{Analysis}

The length of the Pfama1 ectodomain sequence analysed was 1,335 bp (from nucleotide 300 to 1,635, relative to the reference sequence $3 D 7$ [GenBank accession number XM_001347979.1]) and for Pvama1, $1320 \mathrm{bp}$ (from nucleotide 300 to 1620 , relative to the reference sequence Sal-1 [GenBank accession number AF063138]). Differences in length were due to the insertion of five additional residues within Pfama1 DIII (positions 471 to 473, relative to the reference sequence $3 D 7$ ) and the transmembrane region (positions 535 and 540, relative to the reference sequence 3D7). For each of the P. falciparum field isolates, raw sequence data were edited and high quality sequences assembled into contigs using Sequencher version 5.0 [63]. Editing of raw sequence data included trimming the poor quality ends of sequence reads and assembling sequences to the relevant reference sequence in order to generate a consensus sequence. Once assembled to the reference, ambiguous base calls were clarified and if the quality of the chromatogram was not sufficient to enable ambiguous bases to be accurately called, the sample was re-amplified and re-sequenced. Only high quality data was included in the final dataset. Single nucleotide polymorphisms (SNPs) were identified by comparing the consensus sequence for each isolate to the reference strains (P. falciparum 3D7). SNPs were confirmed if they were present in at least one other isolate. Rare SNPs, found in only one isolate, were confirmed by amplifying and sequencing a second independent PCR product. Sequences were deposited in GenBank [KF698984 to KF699059 (Pfama1) and KC702402 to KC702503 (Pvama1)] [51].

Although analysis of the $P$. vivax sequences was previously reported [51], the genomic region investigated in 
the present study was smaller $(1,320 \mathrm{bp})$ and included an additional $96 \mathrm{bp}$ at the 3 ' end not analysed previously in order to enable direct comparison with Pfamal sequences. Hence population genetic analyses were repeated and extended compared to those performed previously for all Pvama1 sequences included in the present study. Nine additional reference sequences were also analysed including the Pfamal sequence of the FVO isolate (GenBank accession no AJ277646.1); six primate-adapted $P$. vivax isolates used for vaccine research (Chesson I, Belem, India VII, Indonesia XIX, Palo Alto and North Korea; GenBank accession nos EU395587 to EU395593) [64].

Multiple alignments were performed using the MUSCLE algorithm implemented in MEGA version 5.0 software [65]. Polymorphism and diversity was estimated using DnaSP version 5.0 [66] by calculating the total number of polymorphic sites $(S)$; synonymous (SP) and non-synonymous (NS) SNPs, average pairwise nucleotide diversity $(\pi)$, number of haplotypes $(h)$ and haplotype diversity $(H d)$, which is an allele frequency based statistic analogous to the heterozygosity and is calculated as follows:

$$
\left.H d=[n /(n-1)]\left[1-\sum\left(f_{i}\right)^{2}\right)\right]
$$

Where $\mathrm{n}$ is the sample size and $f$ is the frequency of the $i^{\text {th }}$ allele [67]. As the number of haplotypes is influenced by sample size, the allelic richness $\left(R_{S}\right)$ was also calculated, as $R_{S}$ is normalized on the basis of the smallest sample size [68]. $R_{S}$ was calculated using FSTAT version 2.9.3 [69]. Additionally, allele frequencies of all amino acid polymorphisms were calculated using CONVERT, version 1.31 [70].

To identify departures from neutrality, a sliding window analysis of Tajima's D was performed using DnaSP version 5.0 [66]. Negative values of Tajima's D indicate an excess of rare alleles consistent with directional selection or recent population expansion whereas positive values indicate an excess of intermediate frequency mutations, suggestive of a recent population bottleneck or balancing selection [71]. Balancing selection maintains alleles at balanced frequencies within populations in order to maintain genetic diversity and therefore an evolutionary advantage under immune pressure.

To estimate the amount of recombination in AMA1 genes, the recombination parameter $(R)$, based on the variance between the average number of nucleotide differences between pairs of sequences, was calculated using DnaSP version 5.0 as follows:

$$
R=4 N r
$$

Where $N$ is the population size and $r$ is the recombination rate per sequence [72]. All parameters of the equation were estimated from the input data by DnaSP software in the process of calculating $R$.
To measure the degree of non-random association between alleles at two or more sites (linkage disequilibrium, LD), $D$ ' [73] and $r^{2}$ [74] were calculated using the 'Full Matrix LD' option of TASSEL software, version 3.0.157 [75]. LD was calculated only for SP and NS SNPs with a minor allele frequency (MAF) or combined MAF $\geq 0.10$ ('common' polymorphisms). 'Combined MAF' refers to the combined frequencies of the minor alleles detected at specific sites. Only 'common' SP and NS SNPs with a MAF or combined MAF $\geq 0.10$ were analysed as inclusion of rare alleles can artificially inflate values of $D^{\prime}$ [76]. Tri-allelic SNPs were split so that the major allele was analysed separately with each of the minor alleles. The Fisher's Exact test was used to measure significance of any associations.

The PvAMA1 model was generated as previously described [51]. The PfAMA1 model was generated using the same protocol [51] with the following modifications. The chimeric template was generated using overlays of the P. vivax (Protein Data Bank ID: 1W81) and P. falciparum (Protein Data Bank ID: 1Z40) AMA1 crystal structures, and grafting PvAMA1 loop residues onto the PfAMA1 core. The grafted residues were as follows: PvAMA1 residues 41 to 52 (numbering relative to the $P$. vivax reference strain Sal-1 AMA1 sequence) which correspond to PfAMA1 residues 96 to 107 (numbering relative to the $P$. falciparum reference strain 3D7 AMA1 sequence); PvAMA1 residues 116 to 123 which correspond to PfAMA1 residues 171 to 178 ; and as the crystal structure of PfAMA1 DIII is yet to be solved, PvAMA1 residues 383 to 400 and 416 to 474 , which correspond to PfAMA1 residues 438 to 455 and 471 to 532 , respectively. Three PfAMA1 loop structures missing from both the PvAMA1 and PfAMA1 crystal structures were generated automatically by Modeller using only stereochemical and geometric restraints: PfAMA1 residues 264 to 274 (loop 1), 383 to 387 (loop 2) and 456 to 470 (loop 3). The P. falciparum $3 D 7$ reference sequence (GenBank accession no XM_001347979.1) was aligned against the chimeric template sequence in order to generate the PfAMA1 model. As the incomplete PfAMA1 crystal structure necessitated modeling three missing loop structures and grafting the entire DIII from the PvAMA1 crystal structure, one hundred PfAMA1 models were generated in order to determine the optimized model with the lowest final probability density function (PDF) energy for structural analysis. Discovery Studio, version 3.1 (Accelrys, San Diego, CA, USA) was used to prepare figures.

\section{Results}

\section{Polymorphism and diversity of Plasmodium falciparum} and Plasmodium vivax AMA1 genes

The total number of polymorphic sites (S) in Pfama1 was almost double that of Pvama1 (Table 1). Similarly, the number of NS SNPs for Pfama1 was nearly double 
Table 1 Estimates of AMA1 genetic diversity for $P$. falciparum and $P$. vivax in PNG

\begin{tabular}{|c|c|c|c|c|c|}
\hline & & \multicolumn{2}{|c|}{ P. falciparum } & \multicolumn{2}{|c|}{ P. vivax } \\
\hline & & Wosera & Madang & Wosera & Madang \\
\hline \multirow{9}{*}{ Whole ectodomain } & $n$ & 44 & 32 & 41 & 61 \\
\hline & $S$ & 56 & 52 & 34 & 36 \\
\hline & $\pi\left(\times 10^{-3}\right)$ & 14.2 & 13.4 & 7.6 & 8.5 \\
\hline & NS & $48^{a}$ & $47^{b}$ & 26 & $29^{c}$ \\
\hline & $\mathrm{SP}$ & $1^{a}$ & $1^{b}$ & 8 & $4^{c}$ \\
\hline & $h$ & 20 & 12 & 38 & 50 \\
\hline & Hd & 0.92 & 0.91 & 0.99 & 0.99 \\
\hline & $R_{S}$ & 19 & 12 & 38 & 45 \\
\hline & $R$ & 45.8 & 36.6 & 70.9 & 66.2 \\
\hline \multirow{6}{*}{$\mathrm{DI}$} & $S$ & 36 & 32 & 21 & 23 \\
\hline & $\pi\left(\times 10^{-3}\right)$ & 25.1 & 24.5 & 15.7 & 17.3 \\
\hline & NS & $30^{\mathrm{a}}$ & $29^{b}$ & 14 & $16^{c}$ \\
\hline & SP & $1^{a}$ & $1^{b}$ & 7 & $4^{c}$ \\
\hline & $h$ & 18 & 12 & 21 & 25 \\
\hline & $H d$ & 0.92 & 0.91 & 0.96 & 0.95 \\
\hline \multirow{6}{*}{ DII } & $S$ & 8 & 8 & 4 & 6 \\
\hline & $\pi\left(\times 10^{-3}\right)$ & 8.3 & 6.6 & 2.3 & 2.6 \\
\hline & NS & 8 & 8 & 4 & 6 \\
\hline & $\mathrm{SP}$ & 0 & 0 & 0 & 0 \\
\hline & $h$ & 10 & 10 & 5 & 9 \\
\hline & Hd & 0.86 & 0.85 & 0.59 & 0.57 \\
\hline \multirow{6}{*}{ DIII } & $S$ & 7 & 7 & 1 & 1 \\
\hline & $\pi\left(\times 10^{-3}\right)$ & 14.8 & 13.7 & 0.2 & 1.3 \\
\hline & NS & 7 & 7 & 1 & 1 \\
\hline & $\mathrm{SP}$ & 0 & 0 & 0 & 0 \\
\hline & $h$ & 9 & 8 & 2 & 2 \\
\hline & $\mathrm{Hd}$ & 0.86 & 0.84 & 0.04 & 0.25 \\
\hline
\end{tabular}

$\mathrm{DI}=$ domain I; DII=domain II; DIII; domain III; $n=$ number of samples; $S=$ number of polymorphic sites; $\pi=$ nucleotide diversity; $N S=$ number of non-synonymous single nucleotide polymorphisms (SNPs); $\mathrm{SP}=$ number of synonymous SNPs; $h=$ number of haplotypes; $H d=$ haplotype diversity; $R_{S}$ : allelic richness; $R$ : recombination parameter.

${ }^{a}$ Complex codons not analysed by DnaSP software: 3 codons $(292,293,294)$ $(301,302,303)(304,305,306)$.

${ }^{\mathrm{b}}$ Complex codons not analysed by DnaSP software: 2 codons $(292,293,294)$ (301, 302, 303).

${ }^{\mathrm{C} C}$ Complex codon not analysed by DnaSP software: 1 codon $(550,551,552)$.

that of Pvama1 (Table 1). Conversely, the number of SP SNPs was much lower in Pfama1 than for Pvama1. Despite fewer polymorphic sites overall, the number of Pvama1 haplotypes was more than four times that of Pfama1 in Madang and nearly double the number detected for Pfama1 in the Wosera. Haplotype diversity was very high for both species, with Pvama1 haplotype diversity close to the maximum of 1 , indicating that all haplotypes are rare (Table 1 ).

To ensure that using a combined $P$. vivax population did not impact estimates of $P$. falciparum diversity, AMA1 diversity within the Mugil $P$. vivax population was also investigated. Diversity of AMA1 within the Mugil P. vivax population was similar to that of the combined Madang population, namely that diversity was high and exceeded that of the Madang (Mugil) P. falciparum population (Additional files 1 and 2). Hence, the differences observed were not due to stratified population effects.

Polymorphism was distributed unevenly among the three domains of AMA1 in both species. The number of SNPs, and consequently nucleotide diversity, peaked in DI for both Pfama1 and Pvama1 (Figures 1A and B; Table 1). Again, there were a greater number of SNPs in Pfama1 DI compared to Pvama1, but a higher number of SP SNPs and increased allelic diversity resulted in higher overall diversity of Pvama1 DI haplotypes (Table 1). In contrast, the diversity of Pfamal exceeded that of Pvama1 in DII and DIII (Figure 1B; Table 1). For DII, the number of haplotypes in Madang was similar for both species, however in Wosera the number of Pfama1 NS SNPs and haplotypes greatly exceeded that of Pvama1 (Table 1). For DIII, there were seven NS SNPs and a peak in nucleotide diversity ( $\pi$ ) for Pfama1 with eight and nine haplotypes in the Wosera and Madang populations, respectively but only a single NS SNP and two haplotypes were detected for Pvama1 in each population (Figure 1B; Table 1). No SP SNPs were detected in DII or DIII for either species.

\section{Evolution of Plasmodium falciparum and Plasmodium vivax AMA1 genes}

To identify departures from neutral evolution, the Tajima's D statistic was calculated using a sliding window approach along the length of the region encoding the AMA1 ectodomain for each population of both species. Although positive values of Tajima's D, most likely due to balancing (immune) selection, were observed along the length of the ectodomain for Pfama1, highly significant positive values of Tajima's D $(p<0.01)$ were observed in DIII (Figure 1C). This suggests that DIII is an important target of protective host immune responses within Pfama1.

For Pvama1, only DI deviated significantly from neutral expectations, suggesting that this region is a strong target of functional host immune responses (Figure 1C). Interestingly, although the results were similar for the Wosera and Madang Pfama1 populations, differences between the Madang and Wosera Pvama1 populations were observed. Whilst significantly positive values $(p<0.05)$ of Tajima's D were observed within DI for both populations, negative values were also observed in DI for the Wosera population, which can indicate an excess of rare alleles at these sites consistent with purifying selection or a recent population expansion. The negative values of Tajima's D did not reach significance (Figure $1 \mathrm{C}$ ). 

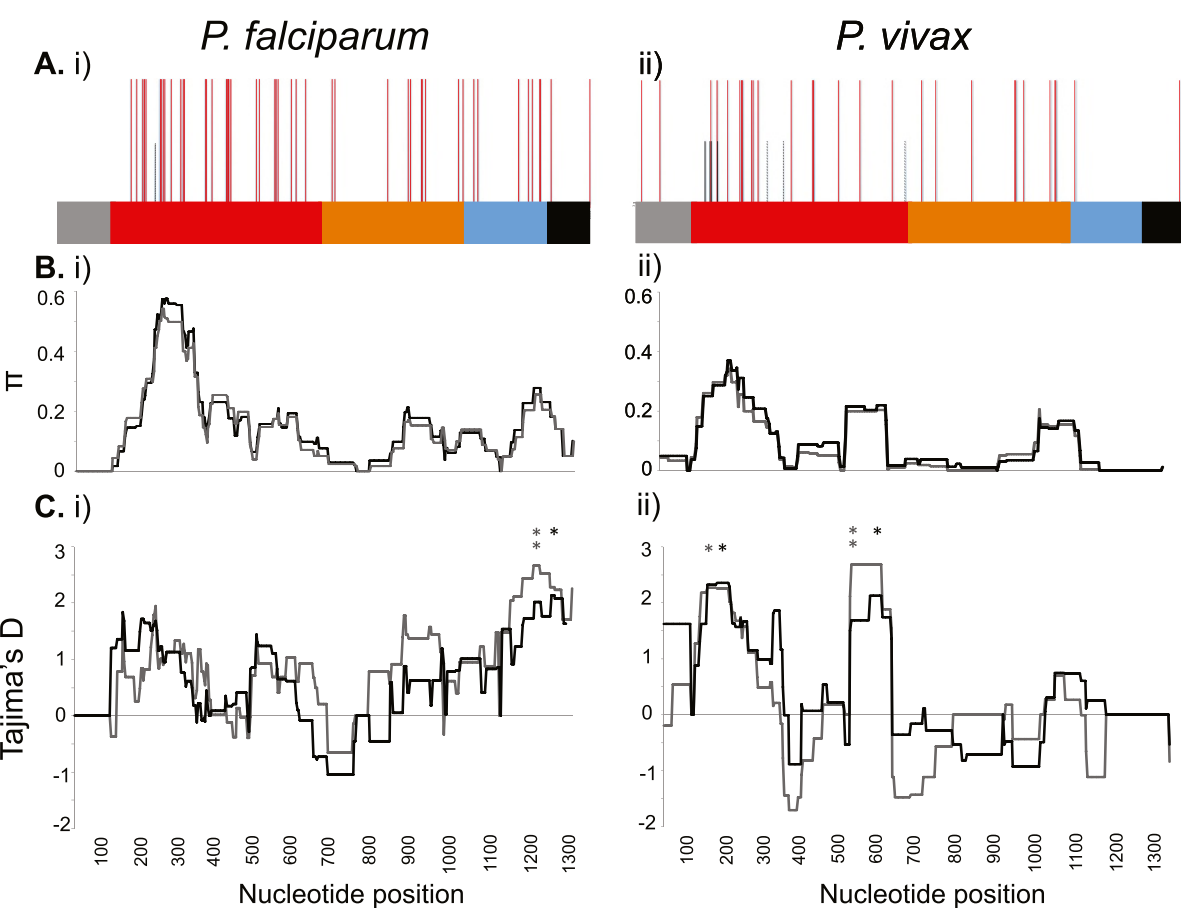

ii)

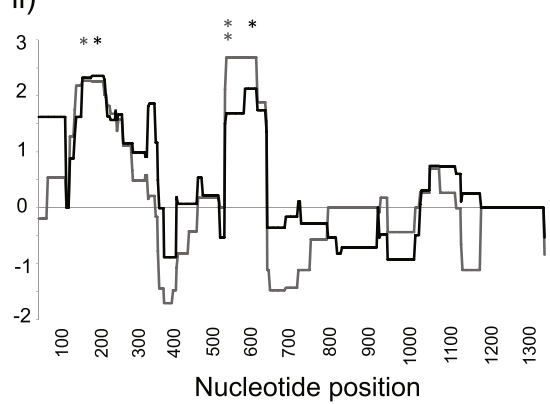

Figure 1 Polymorphism and selection of AMA1 genes in Plasmodium falciparum and Plasmodium vivax populations of Papua New Guinea. The following results are based on the total dataset of 76 P. falciparum and 102 P. vivax sequences A) Polymorphism: Schematic of the (i) P. falciparum and (ii) P. vivax genes encoding the AMA1 ectodomain, with all polymorphisms including non-synonymous (NS SNP, red lines), synonymous (SP SNP, black lines) and singleton (dashed red and black lines, respectively) sites shown. Location of residues is indicated by the colored panel along the top of the chart: signal sequence (grey), DI (red), DII (orange), DIII (blue), transmembrane region (black). B) Nucleotide diversity: Sliding window analysis showing nucleotide diversity ( $\pi$ values for (i) Pfama1 and (ii) Pvama1. A window size of 100 bp and a step size of 3 bp were used. C) Natural selection. Sliding window calculation of Tajima's D was performed for all (i) 76 Pfama1 sequences and (ii) 102 Prama1 sequences (black = Madang; grey =Wosera). A window size of 100 and a step size of 3 were used. A single asterisk (black= Madang; grey $=$ Wosera) indicates significant values for which $p<0.05$; and double asterisk indicates $p<0.01$.

\section{Recombination and linkage disequilibrium (LD) in} Plasmodium falciparum and Plasmodium vivax AMA1 genes To explore how much genetic exchange occurs at the AMA1 locus in each species, the amount of recombination and LD were estimated. The recombination parameter, $R$, was higher for $P$. vivax compared to $P$. falciparum, and was higher in the Wosera compared to Madang for both species (Table 1). Linkage disequilibrium was then estimated between all pairs of 'common' polymorphic SP and NS SNPs (Pfama1, $\mathrm{n}=45 ;$ Pvama1 = 24). Significant $(p<0.0001)$ LD values between distant sites were observed for $P$. falciparum, whereas LD decayed with physical distance between $P$. vivax polymorphic sites (Figure 2). Together, these data demonstrate that the rate of recombination in Pvama1 exceeds that of Pfama1 in these populations across the region evaluated.

\section{Three-dimensional structural modeling of PfAMA1 and PvAMA1 polymorphisms}

All residues found to be polymorphic were mapped to the respective three-dimensional model for $P$. falciparum and P. vivax AMA1. Previously, only PvAMA1 polymorphisms predicted to be under balancing selection were mapped to the $P$. vivax three-dimensional model [51]. In the present study, all polymorphic loci were mapped, extending the previous study by including eight additional sites polymorphic amongst PNG PvAMA1 sequences: M51, D133, A172, G253, R317, T359, K400 and Q484. In order to determine the proximity of the polymorphic residues to the ligand-binding cleft, the residues comprising the hydrophobic cleft [77] were also mapped.

All polymorphic residues (PfAMA1, $\mathrm{n}=47$; PvAMA1, $\mathrm{n}=28$ ) identified amongst PNG isolates that were located within the region used to generate the respective models (Additional file 3) mapped to solvent-exposed surfaces (Figure 3). Consistent with the findings of Bai et al. [33], a biased distribution of polymorphisms was observed for both species, with 41 (87\%) PfAMA1 polymorphic residues and 25 (89\%) PvAMA1 located on one face of the AMA1 molecule. For PfAMA1, three polymorphic DII residues (K376T, H393R and K395R) were located on the opposing 'silent' face (Figure 3; Additional file 4). For PvAMA1, two polymorphic signal sequence residues, M51I and R66K, and one DII residue, T359A, 


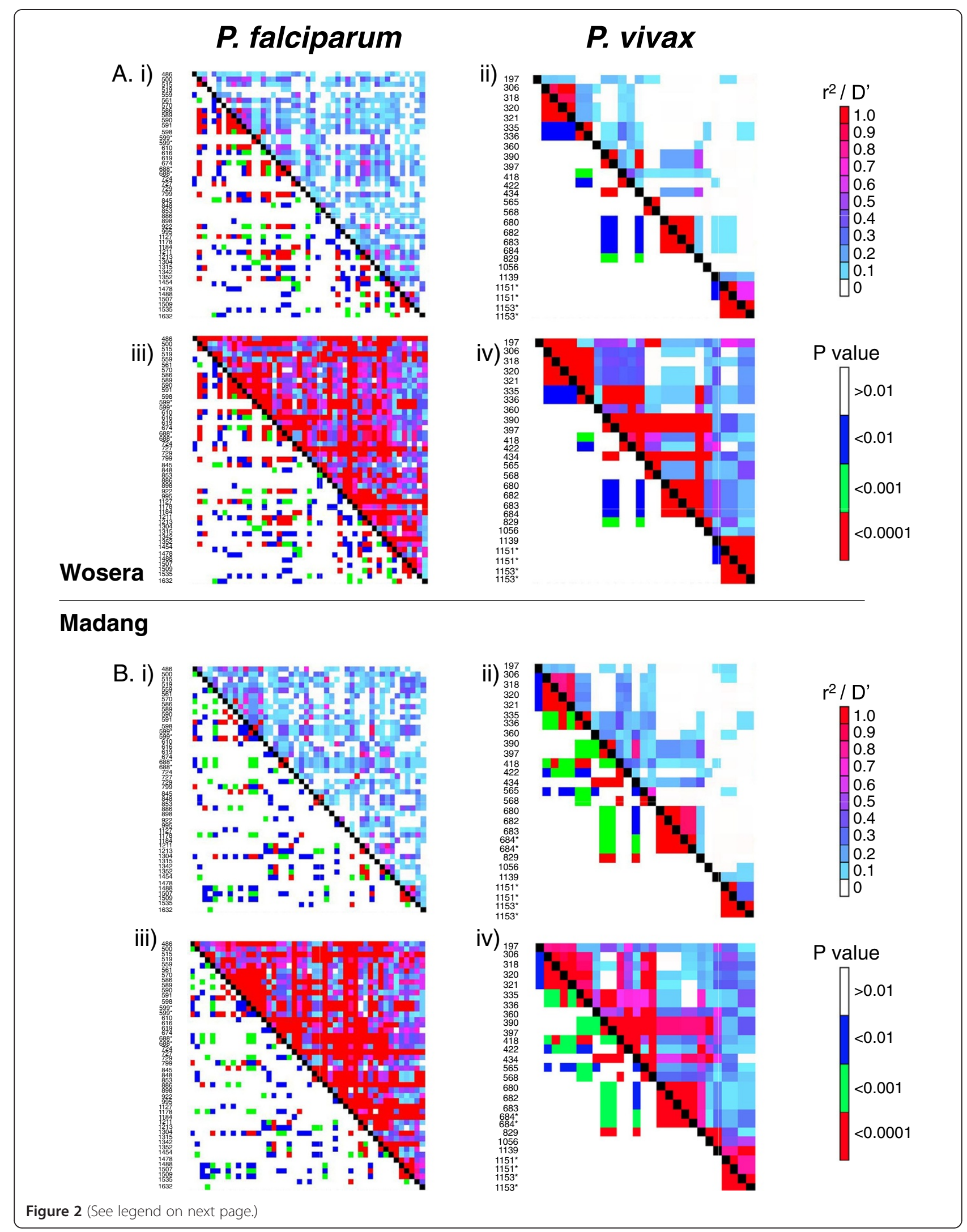


(See figure on previous page.)

Figure 2 Linkage disequilibrium in AMA1 genes of Plasmodium falciparum and Plasmodium vivax populations in Papua New Guinea. The indices of linkage disequilibrium (LD), $r^{2}$ ( $\mathrm{i}$ and ii) and $D^{\prime}$ (iii and iv) were calculated for all AMA1 polymorphisms with a MAF $\geq 0.10$ for (A) Wosera and (B) Madang populations. SNP position is shown on the $Y$ axis and black squares represent self comparisons. Numbering is relative to the Pfama1 3D7 reference sequence (GenBank accession no: XM_001347979.1) and the Pvama1 Sal-1 reference sequence (GenBank accession no: AF063138), respectively. Coloured squares above the black diagonal represent values obtained for each pair of sites following $r^{2}$ or $D^{\prime}$ calculations. Below the black diagonal line on each heat map, coloured squares reflect the significance value ( $p$ ). An asterisk denotes tri-allelic SNPs that were split into two so that the major allele was analysed separately with each of the minor alleles. For interpretation, see the scale to the right of diagrams.

were located on the opposing 'silent' face (Figure 3; Additional file 5).

In both species, Tyr251, the hydrophobic cleft residue reported to be essential for RON2 binding [78] was strictly conserved (Additional file 3 ). Of the 15 orthologous sites that were polymorphic in both species, ten were located within DI and five within DII (Additional files 3, 4 and 5). Eight of those surrounding the hydrophobic binding cleft have been previously associated with antigenic escape for PfAMA1 ([11,79], Additional files 3, 4 and 5).

Whilst the overall distribution of polymorphic residues was similar for both species, important differences with regards to species-specific polymorphisms surrounding and comprising the binding cleft were observed. Two hydrophobic binding cleft residues, M190I and M224I, were found to be polymorphic in 25 and $4 \%$ of PNG PfAMA1 sequences, respectively (Figure 3; Additional files 3 and 4). In all sequences only one of these sites, but not both, was polymorphic. None of the PvAMA1 hydrophobic cleft residues were found to be polymorphic. Additionally, all polymorphic PfAMA1 residues observed amongst PNG sequences that were located proximal to the binding cleft have been associated previously with antigenic escape $[11,79]$. For PvAMA1, with the exception of N130K, all polymorphic residues located proximal to the binding cleft also aligned with PfAMA1 residues belonging to the $\mathrm{c} 1$ or $\mathrm{c} 3$ clusters associated with antigenic escape (Figure 3; Additional file 3). However R317Q, found in three (3\%) PNG PvAMA1 sequences, was located in DII and immediately proximal to the hydrophobic binding cleft (Additional file 5). The orthologous PfAMA1 residue, Ala372, was strictly conserved.

\section{Relevance of PfAMA1 and PvAMA1 diversity to vaccine design}

In order to compare and contrast the extent of PfAMA1 and PvAMA1 antigenic diversity within PNG, haplotypes were constructed using 'common' amino acid polymorphisms (MAF $\geq 0.10$ ), as these polymorphisms are predicted to contribute the majority of antigenic diversity and are therefore relevant to vaccine design [51].

Of 48 PfAMA1 polymorphic amino acid sites, 41 had a MAF, or combined MAF of $\geq 0.10$ (Figure 4A). These 'common' sites included the highly polymorphic $\mathrm{c} 1$ and c1L cluster residues that have been previously associated with antigenic escape [79]. An additional PfAMA1 polymorphic residue at the $\mathrm{C}$-terminal end (position 544) was also included in the haplotype analysis but was not mapped to the PfAMA1 model as it was located outside the region used to generate the crystal structure. Of the 41 'common' PfAMA1 polymorphisms, the majority $(\mathrm{n}=34,83 \%)$ were dimorphic. Five polymorphisms were tri-allelic (12\%) and two were tetra-allelic (5\%) (Figure 4A). The majority of common polymorphisms were clustered in DI ( $\mathrm{n}=25,61 \%$, Figure 4A).

Previously, PvAMA1 polymorphism amongst a population of global sequences was investigated [51]. Whilst it was reported that PNG PvAMA1 sequences were distinct from all others worldwide, as global diversity was the focus of the previous study, the mutations and combinations thereof unique to PNG were not investigated. In the present study, when compared to the 23 sites with a MAF $\geq 0.10$ identified amongst the global $P$. vivax population, three sites (N132, P210, G288) had a MAF $<0.10$ and one site (R438) was invariant amongst PNG sequences. Residue D133, invariant amongst all other populations worldwide [51] had a MAF $\geq 0.10$ amongst PNG sequences. Additionally, seven polymorphic sites with a MAF $<0.10$ amongst the global population were found to be invariant amongst PNG PvAMA1 sequences: G117, D158, H193, V218, K368, V382, N445. Hence, although PvAMA1 diversity was investigated previously, the residues and haplotypes analysed in the present study differ considerably on account of the unique polymorphisms and combinations thereof identified amongst PNG PvAMA1 sequences.

Amongst PNG PvAMA1 sequences, 28 sites were polymorphic however only 18 were common (Additional file 5), less than half the number detected for PfAMA1. PvAMA1 allelic diversity was however higher than of PfAMA1 with a larger proportion of tri-allelic ( $P f: \mathrm{n}=5$, $12 \% ; P v: \mathrm{n}=5,27 \%$ ) sites (Figure 4A). None of the polymorphic PvAMA1 sites were tetra-allelic.

Based on the 41 common PfAMA1 polymorphisms, only 21 PfAMA1 haplotypes were detected (Madang $=12$, Wosera $=18$ ). Despite less than half the number of common amino acid polymorphisms compared to PfAMA1, 
A.

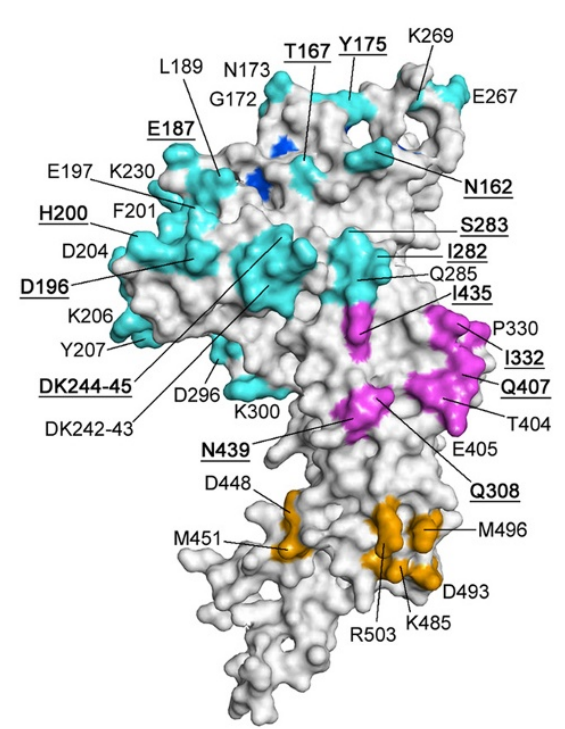

B.

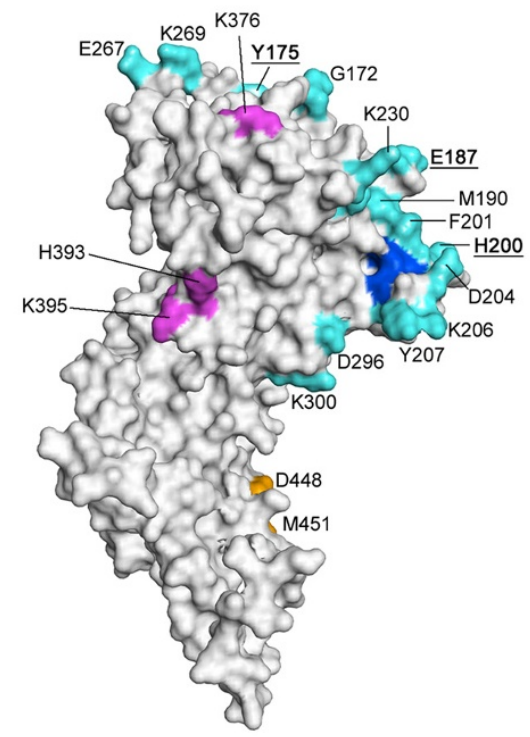

C.

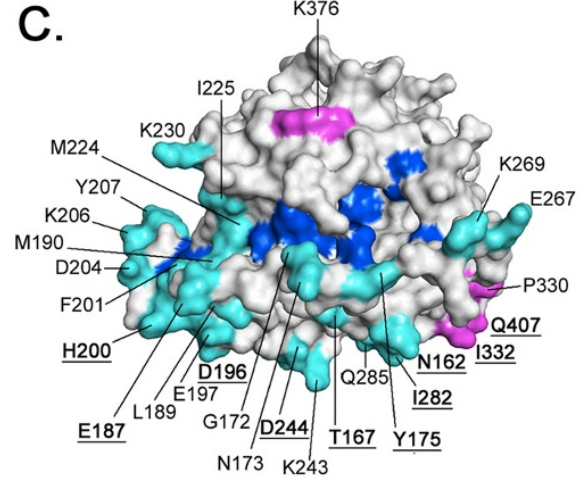

\section{P. vivax}
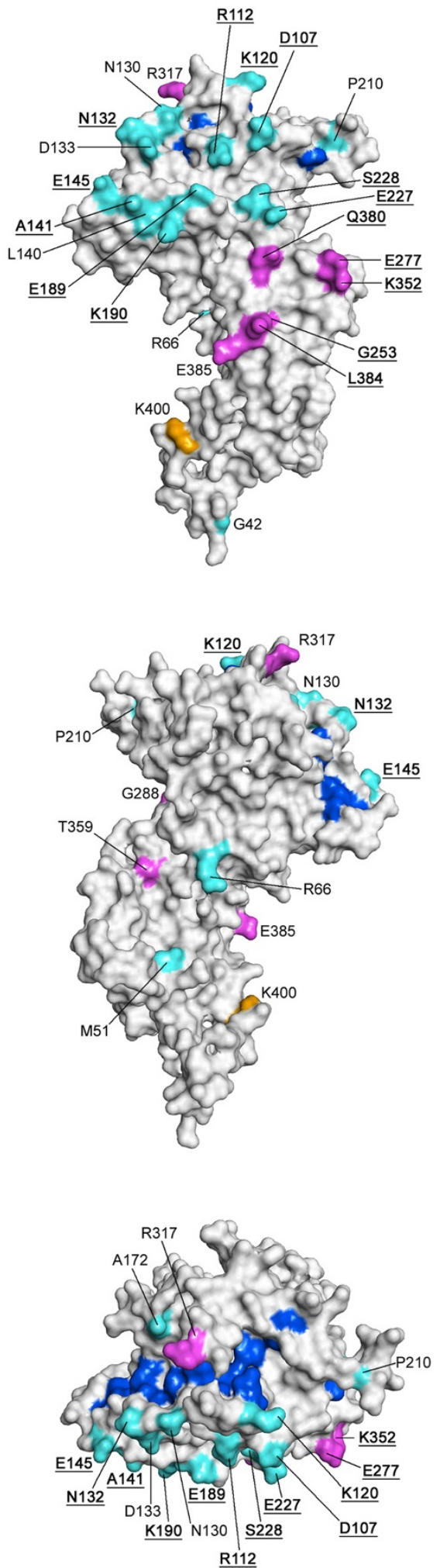

Figure $\mathbf{3}$ (See legend on next page.) 
(See figure on previous page.)

Figure 3 Three-dimensional structural model of Plasmodium falciparum and Plasmodium vivax AMA1 polymorphisms. A) Solvent-accessible surface representation of the 'active face' of the PFAMA1 and PVAMA1 three-dimensional (3D) models. Polymorphic residues are colored according to location: DI in cyan, DII in magenta, DIII in orange. Hydrophobic ligand binding cleft residues are shown in dark blue. Residues labeled with bold, underlined type are polymorphic in both $P$. falciparum and $P$. vivax. Residues of potential immunological relevance with a MAF $\geq 0.10$ are indicated with an asterisk. B) Solvent-accessible surface representation of the 'silent face' of the PfAMA1 and PvAMA1 models. The hydrophobic cleft and polymorphic residues are shown, with coloring and labeling as described for panel A. C) Solvent-accessible surface representation of the PfAMA1 and PVAMA1 models showing a top-view of the hydrophobic binding cleft. Hydrophobic cleft and polymorphic residues are shown, with coloring and labeling as described for panel A. Note that for PfAMA1, hydrophobic cleft residues M224 and M190 are polymorphic and colored cyan (not dark blue) as they are in Dl.

there were 78 unique PvAMA1 haplotypes (Madang $=48$, Wosera $=36)$. Almost half $(47.6 \%, \mathrm{n}=10)$ of the PfAMA1 haplotypes were shared between Madang and Wosera, and four haplotypes were highly prevalent (frequency $=11.3$ to 22.7\%). Conversely, all 78 PvAMA1 haplotypes were relatively rare (frequency $<6.5 \%)$ with only $7.7 \%(n=6)$ shared between Madang and Wosera (Figure 4B). This further demonstrates that in PNG, PfAMA1 diversity is substantially lower than PvAMA1 diversity.

Of direct relevance to vaccine design, the prevalence of alleles being used in vaccine development were then investigated. Whilst the PfAMA1 vaccine allele, FVO was found in both populations, albeit at a low frequency (Madang $=6.2 \%$, Wosera $=2.2 \%$; Figure $3 \mathrm{~B})$, the PfAMA1 vaccine allele $3 \mathrm{D} 7$ and none of the seven primateadapted PvAMA1 alleles used for vaccine development, (Sal-1, Chesson I, Belem, India VII, Indonesia XIX, Palo Alto and North Korea), were detected in either population (Figure 4B).

Polymorphism of residues located within the epitopes of invasion inhibitory antibodies is associated with immune escape $[11,79,80]$. All five of the PfAMA1 c1 cluster residues that are essential for binding of the inhibitory antibody 1F9 were polymorphic (E197G/D/Q, H200D/ L/R, F201L, D204N and I225N; Additional file 4) [77]. It has been reported that any substitution of the PfAMA1 Glu197 completely abrogates 1F9 binding [77]. Only five of the 76 PNG PfAMA1 sequences investigated (6.5\%) had the reference allele at this residue (Additional file 4: Table S1). Six PfAMA1 DII residues (Asp348, Lys351, Gln352, Phe385, Asp388 and Arg389) reported to be critical for binding of the 4G2 inhibitory antibody [81] were conserved amongst the PfAMA1 PNG sequences analysed.

\section{Discussion}

This is the first study of comparative $P$. falciparum and $P$. vivax genetic diversity to be conducted in a setting of high or similar co-endemicity, and the first study to directly compare the diversity of sympatric parasite populations in PNG. Additionally, presented here is the most comprehensive analysis of $P$. falciparum AMA1 diversity in PNG performed to date. Indices of recombination, haplotype number and diversity for PvAMA1 exceeded those of PfAMA1 in both geographic areas investigated. Consistent with previous observations following analysis of neutral molecular markers [53-56], it is likely that the effective population size of $P$. vivax exceeds that of $P$. falciparum within PNG.

For each species, diversity was similar in both geographic areas investigated despite the fact that Wosera had a lower prevalence of $P$. vivax than Madang [54] and a highly variable prevalence of $P$. falciparum among the different villages [55]. In the years prior to sample collection, increased access to treatment and the widespread roll-out and use of insecticide-treated bed nets (ITNs) resulted in a significant decline in the prevalence of both $P$. falciparum and $P$. vivax infection in the Wosera [82]. However, in the same geographic area in the year 2000, 27 PfAMA1 DI haplotypes were identified from 168 samples [35] compared to the 18 haplotypes from only 44 samples collected in 2005 (this study), suggesting that reductions in prevalence have had a limited impact on Pfama1 diversity. Reduced malaria parasite prevalence is a clear indicator that the control measures implemented in the Wosera have been effective. However, further substantial decreases in the prevalence of both species are required within this region to impact parasite antigenic diversity, which has long been a barrier to development of a successful malaria vaccine $[22,83,84]$.

Whilst the overall distribution of polymorphic residues was similar between the species, species-specific differences with regard to the number of polymorphic residues, levels of synonymous polymorphism and substitution of specific AMA1 residues were observed. Consistent with previous observations, the level of synonymous polymorphism amongst Pvamal sequences exceeded that of Pfama1 [48]. Although higher than Pfama1, it has been suggested that synonymous polymorphism in Pvama1 may not be neutral as the level of Pvama1 synonymous polymorphism is greatly reduced in comparison to another $P$. vivax merozoite antigen gene, merozoite surface antigen 1 (Pvmsp1) [52]. In addition, the overall number of polymorphic residues in $P$. falciparum was almost double that of $P$. vivax. Amino acid polymorphisms within 

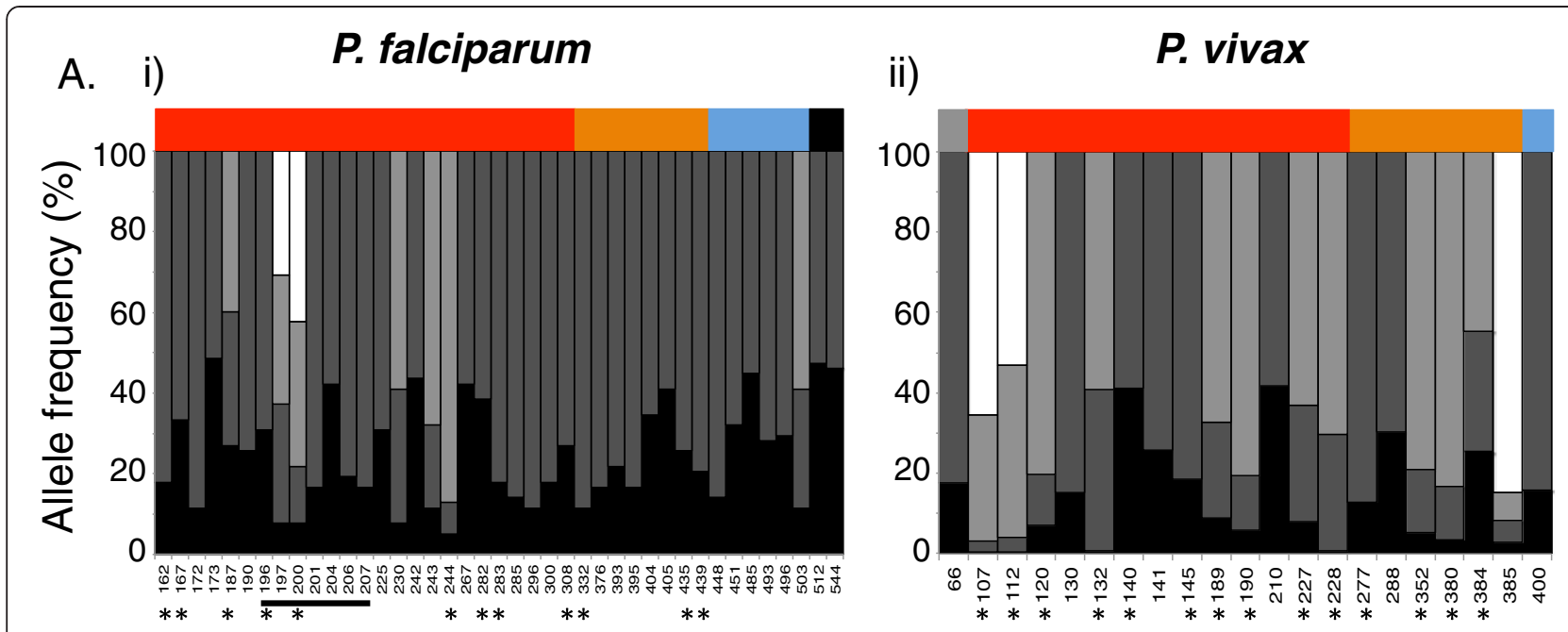

B. i)

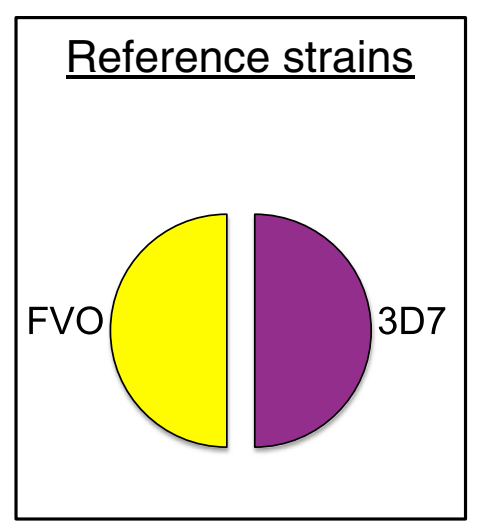

Wosera

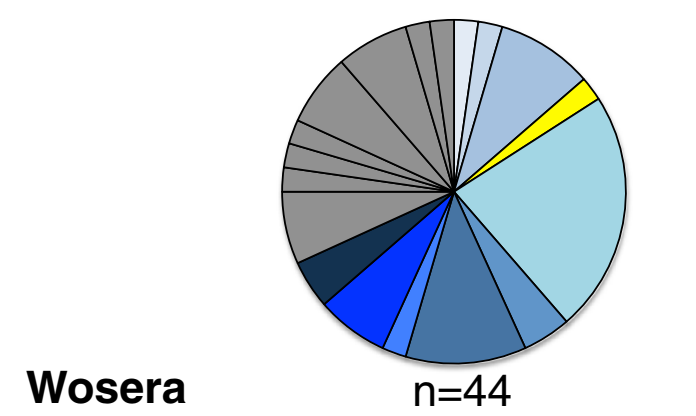

Madang

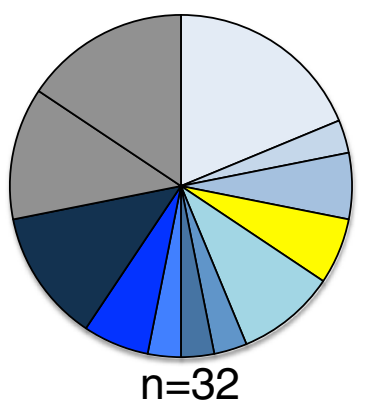

ii)

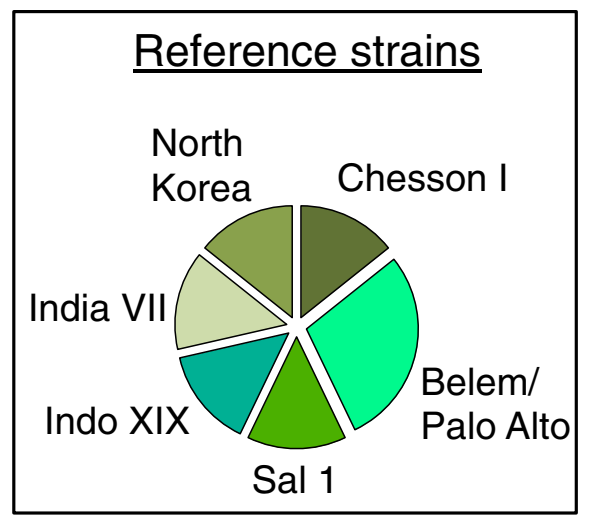

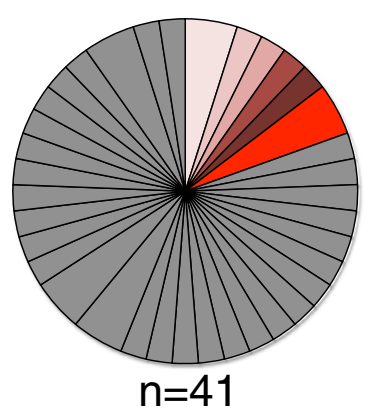

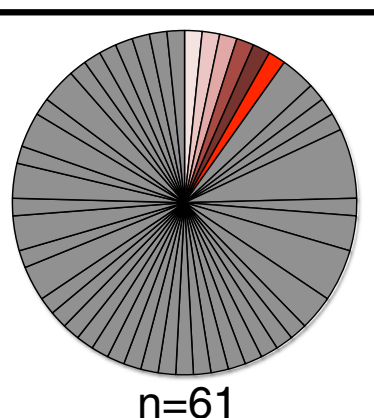

Figure 4 (See legend on next page.) 
(See figure on previous page.)

Figure 4 Frequency of AMA1 polymorphisms and haplotypes in Plasmodium falciparum and Plasmodium vivax populations in Papua New Guinea. A) Polymorphisms. The frequencies of common polymorphisms are shown for (i) PfAMA1 ( $\mathrm{n}=41)$ and (ii) PVAMA1 ( $\mathrm{n}=18$ ). Location of residues is indicated by the colored panel along the top of the chart: signal sequence (grey), DI (red), DII (orange), DIII (blue), transmembrane region (black). Allele frequencies are indicated by the proportion of each bar shaded. Sites that are polymorphic in both species are indicated by an asterisk. Antigenic escape residues defined for PfAMA1 (the "c1L" cluster) are indicated by the horizontal black line (ii). B) Haplotypes. Frequencies of haplotypes based on common polymorphisms for (i) PfAMA1 ( $n=21$ ) and (ii) PvAMA1 ( $\mathrm{n}=78$ ). Coloured segments indicate shared haplotypes between the two populations and grey indicates those unique to one population. The size of the fragment reflects the relative frequency of the haplotype within the population. Reference strain haplotypes are colored to highlight presence/absence in the populations investigated. For PfAMA1, only one haplotype was identical to a reference strain (FVO). No naturally circulating PVAMA1 strains shared haplotypes with any of the reference strains analysed, and thus they are shown in grey. Sample size (n) and origin are indicated.

malarial antigens are typically clustered within sequences coding for B- and T-cell epitopes, hence are associated with immune evasion [85]. Indeed, AMA1 substitutions of potential clinical and functional importance were found in PNG parasites. These included PfAMA1 K230Q/E, which has previously been associated with clinical malaria in Kenyan children and is located at the periphery of the interface between AMA1 and growth-inhibitory antibody 1F9 [36]. Located within or proximal to the hydrophobic binding cleft, the PfAMA1 M190I and M224I, and PvAMA1 R317Q substitutions were also observed. The latter two substitutions have not been previously described, and may play a role in immune evasion, or have functional consequences for binding to RON2 [78]. However, it has been reported that not all genetic diversity within PfAMA1 is antigenically relevant and that indeed it may be overcome with careful selection of a few divergent alleles [80]. Whereas the majority of polymorphic PfAMA1 residues were bi-allelic, $27 \%$ of the PvAMA1 polymorphic residues had three variants, contributing to the higher overall diversity. Whether the same diversity-covering approach can be used to overcome PvAMA1 diversity remains unknown, and studies to identify the specific PvAMA1 residues associated with immune evasion must be performed.

Furthermore, although AMA1 DI was clearly under balancing selection in both species, the strongest signatures of immune selection were detected in DI for $P$. vivax, and DIII for P. falciparum, suggesting that the dominant immune targets may differ between the species. Analysis of 64 Pvama1 sequences from Sri Lanka demonstrated that PvAMA1 DII is also under selection [40]. However, the results of the present study are analogous to those obtained for 372 global PvAMA1 sequences, the largest and most comprehensive analysis of worldwide PvAMA1 diversity performed to date, and suggest that $\mathrm{DI}$ is an immunodominant region of $P v A M A 1$ [51]. That nucleotide diversity and signatures of immune selection for PfAMA1 are highest within DI and DIII, has also been reported following analysis of African P. falciparum sequences $[36,86]$. It has been proposed that strong signatures of selection within PfAMA1 DIII may be due to T-cell responses against allele-specific epitopes [36,87,88]. However, LD between polymorphic DI and DIII residues was also observed amongst PfAMA1, but not PvAMA1, sequences. Previously, LD was reported to be absent among putatively neutral microsatellites in the same sample set [53]. Hence, the LD between PfAMA1 DI and DIII residues might be the result of conformational association between residues comprising important domain-spanning epitopes [36] or alternatively, compensatory mutations to preserve protein function. However, as none of the linked residues co-localized when mapped to the PfAMA1 3D structural model, this is unlikely.

Differences between the two malaria species with regards to levels of polymorphism, diversity, LD and rates of recombination might instead reflect differences in population size and potentially, divergent evolutionary histories of $P$. falciparum and P. vivax within PNG. Recombination accounts for the majority of the diversity observed within malarial antigens [85]. It occurs much more frequently than point mutations resulting in amino acid changes, and can change several nucleotides at once in a single event [85]. As recombination results in production of new variants by combining existing types found within a population, effective population size is a key determinant of population genetic diversity [76,89]. Genome-wide sequencing of a small panel of geographically diverse isolates has demonstrated that the worldwide genetic diversity and effective population size of $P$. falciparum is greatly reduced compared to $P$. vivax, due to at least one major population bottleneck and multiple drug-induced selective-sweeps [8]. Despite also experiencing strong drug pressure, the greater stability of the larger, more diverse and ancient global $P$. vivax population can be attributed to the latent hypnozoite reservoir and more rapid rate of gametogenesis [8]. During past malaria elimination attempts in PNG, large-scale malaria control programs carried out from the 1950s-70s may have initially had a greater impact on P. falciparum until the emergence of chloroquine-resistant strains [90,91]. In the 1980 s, chloroquine-resistant $P$. vivax also emerged 
in PNG [92,93], although the drug pressure on this parasite is not expected to be as strong as that on P. falciparum due to the lower frequency of clinical cases and antimalarial treatment. Analysis of neutral microsatellite markers has shown that $P$. falciparum populations of Madang and Wosera are genetically differentiated [53], however very limited population structure is observed in Pfama1. This might be the result of past reduction in effective population size (e g, a bottleneck), after which common AMA1 haplotypes would be maintained by balancing selection while differentiation at neutral markers would increase through genetic drift. In contrast, very weak $P$. vivax population structure [56] and detection of a high number of extremely diverse, low frequency PvAMA1 haplotypes is consistent with a stable and large effective population size, high levels of recombination and high gene flow between areas. Divergent values of Tajima's D for Pvamal sequences from the Wosera and not Madang potentially suggests that the environmental or selective pressures acting on the Madang and Wosera $P$. vivax populations may not be the same (i. e. local adaptation). Combined, it is therefore likely that in PNG, Pvama1 diversity exceeds that of Pfama1 on account of a larger, more frequently recombining and more ancient $P$. vivax population compared to $P$. falciparum. However, as microsatellites are highly polymorphic $[12,59]$ and AMA1 is under selection in both species [36,51], it will be necessary to analyse more slowly evolving loci, such as mitochondrial DNA in order to clarify the evolutionary histories of $P$. falciparum and $P$. vivax in PNG.

\section{Conclusions}

It is likely that the diversity of PvAMA1 far exceeds that of PfAMA1 in a highly endemic region of PNG because of the underlying parasite population biology [53,94]. Diversity was extremely high in both species and in both populations investigated, despite recent reductions in prevalence in one area (Wosera). Hence, far greater reductions in prevalence of both species will be required to impact the diversity of $P$. falciparum and $P$. vivax AMA1. Differing patterns of balancing selection suggest that dominant immune targets may differ between the two species, which is important knowledge for development of AMA1-based vaccines. Furthermore, vaccine haplotypes for both species were found to circulate at very low frequencies if at all, in PNG. As inclusion of alleles not representative of a given population may result in poor vaccine efficacy [80], this important observation suggests that vaccines based on reference strains might have limited efficacy in this region. The results of this study highlight differences in the population biology and evolution of $P$. falciparum and $P$. vivax in PNG, and have important implications for the design of AMA1based vaccines.

\section{Additional files}

\begin{abstract}
Additional file 1: Frequency of AMA1 polymorphisms and haplotypes in the Plasmodium falciparum and Plasmodium vivax Madang and Mugil populations of Papua New Guinea. i) Haplotypes. Frequencies of haplotypes based on common polymorphisms for (A) PvAMA1 Madang ( $\mathrm{n}=18)$, (B) PvAMA1 Mugil $(\mathrm{n}=18)$ and (C) PfAMA1 $(n=41)$. Coloured segments indicate shared haplotypes between the Wosera and Madang/Mugil populations for each species. Grey indicates haplotypes unique to the specified population. For PfAMA1, only one haplotype was identical to a reference strain (yellow: FVO). No naturally circulating PVAMA1 strains shared haplotypes with any of the reference strains analysed. Sample size (n) and origin are indicated. ii) Polymorphisms. The frequencies of common polymorphisms are shown for (A) PVAMA1 Madang ( $n=18)$, (B) PvAMA1 Mugil $(n=18)$ and (C) PfAMA1 $(n=41)$ Location of residues is indicated by the colored panel along the top of the chart: signal sequence (grey), DI (red), DIl (orange), DIII (blue), transmembrane region (black). Allele frequencies are indicated by the proportion of each bar shaded.
\end{abstract}

Additional file 2: Estimates of AMA1 genetic diversity for Plasmodium falciparum and Plasmodium vivax within Madang and Mugil.

Additional file 3: Protein sequence alignment showing regions of Plasmodium falciparum and Plasmodium vivax AMA1 used to generate three-dimensional models. The P. falciparum 3D7 (GenBank accession no: XM_001347979) and P. vivax Sal-1 (GenBank accession no: AF063138) reference sequences were aligned using MEGA version 5.0 [65]. Numbers indicate the position of residues relative to those of the P. falciparum sequence. Gaps are indicated by dashes. Red bold type indicates residues observed to be polymorphic in both species; black bold type indicates residues polymorphic in either $P$. falciparum or $P$. vivax. The domain boundaries are demarcated by vertical lines, as indicated. Boxes indicate the positions of antigenic $P$. falciparum amino acid clusters, c1-3 [79]; grey shading indicates antigenic escape residues in the c1L cluster [11].

Additional file 4: Summary of polymorphisms in PfAMA1 sequences from Papua New Guinea. All 47 residues polymorphic amongst PNG PFAMA1 sequences are summarized here. The amino acid position (relative to reference isolate 3D7), the 3D7 allele, the variant allele(s) observed, the number of sequences identical to 3D7 at each polymorphic site and the number of sequences containing the variant allele(s) at each polymorphic site are listed. Sites identical to the 3D7 sequence are indicated by a dot. The 41 polymorphisms with a minor allele frequency (MAF) $\geq 0.10$ are shown in bold. Sites polymorphic in both species are highlighted in red.

Additional file 5: Summary of polymorphisms in PvAMA1 sequences from Papua New Guinea. All 28 residues polymorphic amongst PNG PVAMA1 sequences are summarized here. The amino acid position (relative to reference isolate Sal-1), the Sal-1 allele, the variant allele(s) observed, the number of sequences identical to Sal-1 at each polymorphic site and the number of sequences containing the variant allele(s) at each polymorphic site are listed. Sites identical to the Sal-1 sequence are indicated by a dot. The 18 polymorphisms with a minor allele frequency (MAF) $\geq 0.10$ are shown in bold. Sites polymorphic in both species are highlighted in red.

\section{Competing interests}

The authors declare that they have no competing interests.

\section{Authors' contributions}

AEB and JCR conceived and designed the experiments. AA and JW performed the experiments. AA, AEB and PAR analysed the data. IM and PAR contributed reagents/materials/analysis tools. AA, AEB, JCR, and PAR wrote the paper. PS provided logistical support. All authors read and approved the final manuscript.

\section{Acknowledgements}

The authors would like to thank the volunteers of the communities involved in the study and the staff of the Papua New Guinea Institute of Medical Research. In particular we would like to thank L Tavul for logistical support, J 
Nale and B Kiniboro for collection of field samples, O Toporua for DNA extractions and L Schultz for sample genotyping.

The research was supported by Project Grants 488221 and 1003825 from the National Health and Medical Research Council of Australia (NHMRC). Samples were collected as part of a Bill and Melinda Gates Foundation funded project. IM is a Senior Research Fellow of the NHMRC. This work was made possible through Victorian State Government Operational Infrastructure Support and Australian Government NHMRC IRIISS received by the Burnet Institute and the Walter and Eliza Hall Institute.

\section{Author details}

${ }^{1}$ Centre for Biomedical Research, Burnet Institute, Melbourne, Australia. ${ }^{2}$ Barcelona Centre for International Health Research, Barcelona, Spain. ${ }^{3}$ Division of Infection and Immunity, Walter and Eliza Hall Institute of Medical Research, Melbourne, Australia. ${ }^{4}$ Department of Medical Biology, University of Melbourne, Parkville, Australia. ${ }^{5}$ Department of Immunology, Monash University, Melbourne, Australia. ${ }^{6}$ Department of Surgery Austin Health، University of Melbourne, Heidelberg, Australia. 'School of Biomedical Sciences, CHIRI Biosciences, Faculty of Health Sciences, Curtin University, Perth, Australia. ${ }^{8}$ Papua New Guinea Institute for Medical Research, Goroka, Papua New Guinea. ${ }^{9}$ Centre for Population Health, Burnet Institute, Melbourne, Australia. ${ }^{10}$ Department of Epidemiology and Preventative Medicine, Monash University, Melbourne, Australia. ${ }^{11}$ Current affiliations: Division of Infection and Immunity, Walter and Eliza Hall Institute of Medica Research, Melbourne, Australia. ${ }^{12}$ Current affiliations: Department of Medical Biology, University of Melbourne, Parkville, Australia.

Received: 21 March 2014 Accepted: 22 May 2014

Published: 14 June 2014

\section{References}

1. Genton B, D'Acremont V, Rare L, Baea K, Reeder JC, Alpers MP, Muller I: Plasmodium vivax and mixed infections are associated with severe malaria in children: a prospective cohort study from Papua New Guinea. PLoS Med 2008, 5:e127.

2. Price RN, Tjitra E, Guerra CA, Yeung S, White NJ, Anstey NM: Vivax malaria: neglected and not benign. Am J Trop Med Hyg 2007, 77:79-87.

3. Barcus MJ, Basri H, Picarima H, Manyakori C, Sekartuti, Elyazar I, Bangs MJ, Maguire JD, Baird JK: Demographic risk factors for severe and fatal vivax and falciparum malaria among hospital admissions in northeastern Indonesian Papua. Am J Trop Med Hyg 2007, 77:984-991.

4. Tjitra E, Anstey NM, Sugiarto P, Warikar N, Kenangalem E, Karyana M, Lampah DA, Price RN: Multidrug-resistant Plasmodium vivax associated with severe and fatal malaria: a prospective study in Papua. Indonesia PLOS Med 2008, 5:e128.

5. Guerra CA, Howes RE, Patil AP, Gething PW, Van Boeckel TP, Temperley WH, Kabaria CW, Tatem AJ, Manh BH, Elyazar IR, Baird JK, Snow RW, Hay SI: The international limits and population at risk of Plasmodium vivax transmission in 2009. PLoS Negl Trop Dis 2010, 4:e774.

6. Cotter C, Sturrock HJ, Hsiang MS, Liu J, Phillips AA, Hwang J, Gueye CS, Fullman N, Gosling RD, Feachem RG: The changing epidemiology of malaria elimination: new strategies for new challenges. Lancet 2013, 382:900-911.

7. White NJ, Imwong M: Relapse. Adv Parasitol 2012, 80:113-150

8. Neafsey DE, Galinsky K, Jiang RH, Young L, Sykes SM, Saif S, Gujja S, Goldberg JM, Young S, Zeng Q, Chapman SB, Dash AP, Anvikar AR, Sutton PL, Birren BW, Escalante AA, Barnwell JW, Carlton JM: The malaria parasite Plasmodium vivax exhibits greater genetic diversity than Plasmodium falciparum. Nat Genet 2012, 44:1046-1050.

9. Khim N, Benedet C, Kim S, Kheng S, Siv S, Leang R, Lek S, Muth S, Chea N, Chuor CM, Duong S, Kerleguer A, Tor P, Chim P. Canier L, Witkowski B, Taylor WR, Menard D: G6PD deficiency in Plasmodium falciparum and Plasmodium vivax malaria-infected Cambodian patients. Malar J 2013, 12:171.

10. Orjuela-Sanchez P, Sa JM, Brandi MC, Rodrigues PT, Bastos MS, Amaratunga C, Duong S, Fairhurst RM, Ferreira MU: Higher microsatellite diversity in Plasmodium vivax than in sympatric Plasmodium falciparum populations in Pursat, Western Cambodia. Exp Parasitol 2013, 134:318-326.

11. Takala SL, Coulibaly D, Thera MA, Batchelor AH, Cummings MP, Escalante AA, Ouattara A, Traore K, Niangaly A, Djimde AA, Doumbo OK, Plowe CV: Extreme polymorphism in a vaccine antigen and risk of clinical malaria: implications for vaccine development. Sci Transl Med 2009, 1:2ra5.
12. Anderson TJ, Haubold B, Williams JT, Estrada-Franco JG, Richardson L, Mollinedo R, Bockarie M, Mokili J, Mharakurwa S, French N, Whitworth J, Velez ID, Brockman AH, Nosten F, Ferreira MU, Day KP: Microsatellite markers reveal a spectrum of population structures in the malaria parasite Plasmodium falciparum. Mol Biol Evol 2000, 17:1467-1482.

13. Tanabe K, Mita T, Jombart T, Eriksson A, Horibe S, Palacpac N, Ranford-Cartwright L, Sawai H, Sakihama N, Ohmae H, Nakamura M, Ferreira MU, Escalante AA, Prugnolle F, Bjorkman A, Farnert A, Kaneko A Horii T, Manica A, Kishino H, Balloux F: Plasmodium falciparum accompanied the human expansion out of Africa. Curr Biol 2010, 20:1283-1289.

14. Ferreira MU, Karunaweera ND, da Silva-Nunes M, da Silva NS, Wirth DF, Hartl DL: Population structure and transmission dynamics of Plasmodium vivax in rural Amazonia. J Infect Dis 2007, 195:1218-1226.

15. Imwong M, Nair S, Pukrittayakamee S, Sudimack D, Williams JT, Mayxay M, Newton PN, Kim JR, Nandy A, Osorio L, Carlton JM, White NJ, Day NP, Anderson TJ: Contrasting genetic structure in Plasmodium vivax populations from Asia and South America. Int J Parasitol 2007 37:1013-1022

16. Taylor JE, Pacheco MA, Bacon DJ, Beg MA, Machado RL, Fairhurst RM, Herrera S, Kim JY, Menard D, Povoa MM, Villegas L, Mulyanto, Snounou G, Cui L, Zeyrek FY, Escalante AA: The evolutionary history of Plasmodium vivax as inferred from mitochondrial genomes: parasite genetic diversity in the Americas. Mol Biol Evol 2013, 30:2050-2064.

17. Prugnolle F, Rougeron V, Becquart P, Berry A, Makanga B, Rahola N, Arnathau C, Ngoubangoye B, Menard S, Willaume E, Ayala FJ, Fontenille D, Ollomo B, Durand P, Paupy C, Renaud F: Diversity, host switching and evolution of Plasmodium vivax infecting African great apes. Proc Natl Acad Sci USA 2013, 110:8123-8128.

18. Liu W, Li Y, Shaw KS, Learn GH, Plenderleith $\sqcup$, Malenke JA, Sundararaman SA, Ramirez MA, Crystal PA, Smith AG, Bibollet-Ruche F, Ayouba A, Locatelli S, Esteban A, Mouacha F, Guichet E, Butel C, Ahuka-Mundeke S, Inogwabini BI, Ndjango JB, Speede S, Sanz CM, Morgan DB, Gonder MK, Kranzusch PJ, Walsh PD, Georgiev AV, Muller MN, Piel AK, Stewart FA, et al:: African origin of the malaria parasite Plasmodium vivax. Nat Commun 2014, 5:3346.

19. Arnott A, Barry AE, Reeder JC: Understanding the population genetics of Plasmodium vivax is essential for malaria control and elimination. Malar J 2012, 11:14.

20. Schwartz L, Brown GV, Genton B, Moorthy VS: A review of malaria vaccine clinical projects based on the WHO rainbow table. Malar J 2012, 11:11.

21. Barry AE, Schultz L, Buckee CO, Reeder JC: Contrasting population structures of the genes encoding ten leading vaccine-candidate antigens of the human malaria parasite, Plasmodium falciparum. PLoS One 2009, 4:e8497

22. Takala SL, Plowe CV: Genetic diversity and malaria vaccine design, testing and efficacy: preventing and overcoming 'vaccine resistant malaria'. Parasite Immunol 2009, 31:560-573.

23. Arevalo-Herrera M, Chitnis C, Herrera S: Current status of Plasmodium vivax vaccine. Hum Vaccin 2010, 6:124-132.

24. Pacheco MA, Poe AC, Collins WE, Lal AA, Tanabe K, Kariuki SK, Udhayakumar V Escalante AA: A comparative study of the genetic diversity of the $42 \mathrm{kDa}$ fragment of the merozoite surface protein 1 in Plasmodium falciparum and P. vivax. Infect Genet Evol 2007, 7:180-187.

25. Chenet SM, Pacheco MA, Bacon DJ, Collins WE, Barnwell JW, Escalante AA The evolution and diversity of a low complexity vaccine candidate, merozoite surface protein 9 (MSP-9), in Plasmodium vivax and closely related species. Infect Genet Evol 2013, 20:239-248.

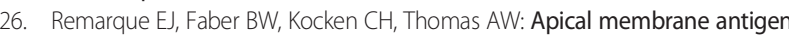
1: a malaria vaccine candidate in review. Trends Parasitol 2008, 24:74-84

27. Gentil F, Bargieri DY, Leite JA, Francoso KS, Patricio MB, Espindola NM, Vaz AJ, Palatnik-de-Sousa CB, Rodrigues MM, Costa FT, Soares IS: A recombinant vaccine based on domain II of Plasmodium vivax Apical Membrane Antigen 1 induces high antibody titres in mice. Vaccine 2010, 28:6183-6190

28. Moon SU, Na BK, Kang JM, Kim JY, Cho SH, Park YK, Sohn WM, Lin K, Kim TS: Genetic polymorphism and effect of natural selection at domain I of apical membrane antigen-1 (AMA-1) in Plasmodium vivax isolates from Myanmar. Acta Trop 2010, 114:71-75

29. Mardani A, Keshavarz H, Heidari A, Hajjaran H, Raeisi A, Khorramizadeh MR Genetic diversity and natural selection at the domain I of apical membrane antigen-1 (AMA-1) of Plasmodium falciparum in isolates from Iran. Exp Parasitol 2012, 130:456-462. 
30. Galinski MR, Barnwell JW: Plasmodium vivax: who cares? Malar J 2008, 7(Suppl 1):S9.

31. Sheehy SH, Duncan CJ, Elias SC, Biswas S, Collins KA, O'Hara GA, Halstead FD, Ewer KJ, Mahungu T, Spencer AJ, Miura K, Poulton ID, Dicks MD, Edwards NJ, Berrie E, Moyle S, Colloca S, Cortese R, Gantlett K, Long CA, Lawrie AM, Gilbert SC, Doherty T, Nicosia A, Hill AV, Draper SJ: Phase la clinical evaluation of the safety and immunogenicity of the Plasmodium falciparum blood-stage antigen AMA1 in ChAd63 and MVA vaccine vectors. PLoS One 2012, 7:e31208.

32. Biswas S, Dicks MD, Long CA, Remarque EJ, Siani L, Colloca S, Cottingham MG, Holder AA, Gilbert SC, Hill AV, Draper SJ: Transgene optimization, immunogenicity and in vitro efficacy of viral vectored vaccines expressing two alleles of Plasmodium falciparum AMA1. PLoS One 2011, 6:e20977.

33. Bai T, Becker M, Gupta A, Strike P, Murphy VJ, Anders RF, Batchelor AH: Structure of AMA1 from Plasmodium falciparum reveals a clustering of polymorphisms that surround a conserved hydrophobic pocket. Proc Natl Acad Sci USA 2005, 102:12736-12741.

34. Yap A, Azevedo MF, Gilson PR, Weiss GE, O'Neill MT, Wilson DW, Crabb BS, Cowman AF: Conditional expression of apical membrane antigen 1 in Plasmodium falciparum shows it is required for erythrocyte invasion by merozoites. Cell Microbiol 2014, 16:642-656.

35. Cortes A, Mellombo M, Mueller I, Benet A, Reeder JC, Anders RF: Geographical structure of diversity and differences between symptomatic and asymptomatic infections for Plasmodium falciparum vaccine candidate AMA1. Infect Immun 2003, 71:1416-1426.

36. Osier FH, Weedall GD, Verra F, Murungi L, Tetteh KK, Bull P, Faber BW, Remarque E, Thomas A, Marsh K, Conway DJ: Allelic diversity and naturally acquired allele-specific antibody responses to Plasmodium falciparum apical membrane antigen 1 in Kenya. Infect Immun 2010, 78:4625-4633

37. Cortes A, Mellombo M, Masciantonio R, Murphy VJ, Reeder JC, Anders RF: Allele specificity of naturally acquired antibody responses against Plasmodium falciparum apical membrane antigen 1. Infect Immun 2005, 73:422-430

38. Mugyenyi CK, Elliott SR, McCallum FJ, Anders RF, Marsh K, Beeson JG: Antibodies to polymorphic invasion-inhibitory and non-inhibitory epitopes of Plasmodium falciparum apical membrane antigen 1 in human malaria. PLoS One 2013, 8:e68304.

39. Mufalo BC, Gentil F, Bargieri DY, Costa FT, Rodrigues MM, Soares IS: Plasmodium vivax apical membrane antigen-1: comparative recognition of different domains by antibodies induced during natural human infection. Microbes Infect 2008, 10:1266-1273.

40. Dias S, Somarathna M, Manamperi A, Escalante AA, Gunasekera AM, Udagama PV: Evaluation of the genetic diversity of domain II of Plasmodium vivax Apical Membrane Antigen 1 (PvAMA-1) and the ensuing strain-specific immune responses in patients from Sri Lanka. Vaccine 2011, 29:7491-7504.

41. Rodrigues MH, Rodrigues KM, Oliveira TR, Comodo AN, Rodrigues MM, Kocken $\mathrm{CH}$, Thomas AW, Soares IS: Antibody response of naturally infected individuals to recombinant Plasmodium vivax apical membrane antigen-1. Int J Parasitol 2005, 35:185-192.

42. Barbedo MB, Ricci R, Jimenez MC, Cunha MG, Yazdani SS, Chitnis CE, Rodrigues MM, Soares IS: Comparative recognition by human IgG antibodies of recombinant proteins representing three asexual erythrocytic stage vaccine candidates of Plasmodium vivax. Mem Inst Oswaldo Cruz 2007, 102:335-339.

43. Hodder AN, Crewther PE, Anders RF: Specificity of the protective antibody response to apical membrane antigen 1. Infect Immun 2001, 69:3286-3294.

44. Kennedy MC, Wang J, Zhang Y, Miles AP, Chitsaz F, Saul A, Long CA, Miller LH Stowers AW: In vitro studies with recombinant Plasmodium falciparum apical membrane antigen 1 (AMA1): production and activity of an AMA1 vaccine and generation of a multiallelic response. Infect Immun 2002, 70:6948-6960.

45. Kocken CH, Withers-Martinez C, Dubbeld MA, van der Wel A, Hackett F, Valderrama A, Blackman MJ, Thomas AW: High-level expression of the malaria blood-stage vaccine candidate Plasmodium falciparum apical membrane antigen 1 and induction of antibodies that inhibit erythrocyte invasion. Infect Immun 2002, 70:4471-4476.

46. Dodoo D, Atuguba F, Bosomprah S, Ansah NA, Ansah P, Lamptey H, Egyir B, Oduro AR, Gyan B, Hodgson A, Koram KA: Antibody levels to multiple malaria vaccine candidate antigens in relation to clinical malaria episodes in children in the Kasena-Nankana district of Northern Ghana. Malar J 2011, 10:108

47. Stanisic DI, Richards JS, McCallum FJ, Michon P, King CL, Schoepflin S, Gilson PR, Murphy VJ, Anders RF, Mueller I, Beeson JG: Immunoglobulin G subclass-specific responses against Plasmodium falciparum merozoite antigens are associated with control of parasitemia and protection from symptomatic illness. Infect Immun 2009, 77:1165-1174.

48. Ord RL, Tami A, Sutherland CJ: ama1 genes of sympatric Plasmodium vivax and P. falciparum from Venezuela differ significantly in genetic diversity and recombination frequency. PLOS One 2008, 3:e3366.

49. Mueller I, Kaiok J, Reeder JC, Cortes A: The population structure of Plasmodium falciparum and Plasmodium vivax during an epidemic of malaria in the Eastern Highlands of Papua New Guinea. Am J Trop Med Hyg 2002, 67:459-464.

50. Mueller I, Genton B, Betuela I, Alpers MP: Vaccines against malaria: perspectives from Papua New Guinea. Hum Vaccin 2010, 6:17-20.

51. Arnott A, Mueller I, Ramsland PA, Siba PM, Reeder JC, Barry AE: Global population structure of the genes encoding the malaria vaccine candidate, Plasmodium vivax apical membrane antigen 1 (PVAMA1). PLoS Negl Trop Dis 2013, 7:e2506.

52. Figtree M, Pasay CJ, Slade R, Cheng Q, Cloonan N, Walker J, Saul A: Plasmodium vivax synonymous substitution frequencies, evolution and population structure deduced from diversity in AMA 1 and MSP 1 genes. Mol Biochem Parasitol 2000, 108:53-66.

53. Schultz L, Wapling J, Mueller I, Ntsuke PO, Senn N, Nale J, Kiniboro B, Buckee CO, Tavul L, Siba PM, Reeder JC, Barry AE: Multilocus haplotypes reveal variable levels of diversity and population structure of Plasmodium falciparum in Papua New Guinea, a region of intense perennial transmission. Malar J 2010, 9:336

54. Arnott A, Barnadas C, Senn N, Siba P, Mueller I, Reeder JC, Barry AE: High genetic diversity of Plasmodium vivax on the north coast of Papua New Guinea. Am J Trop Med Hyg 2013, 89:188-194.

55. Barry AE, Schultz L, Senn N, Nale J, Kiniboro B, Siba PM, Mueller I, Reeder JC: High levels of genetic diversity of Plasmodium falciparum populations in Papua New Guinea despite variable infection prevalence. Am J Trop Med Hyg 2013, 88:718-725.

56. Koepfli C, Timinao L, Antao T, Barry AE, Siba P, Mueller I, Felger I: A large reservoir and little population structure in the South Pacific. PLoS One 2013, 8:e66041.

57. Pinard R, de Winter A, Sarkis GJ, Gerstein MB, Tartaro KR, Plant RN, Egholm M, Rothberg JM, Leamon JH: Assessment of whole genome amplification-induced bias through high-throughput, massively parallel whole genome sequencing. BMC Genomics 2006, 7:216.

58. Havryliuk T, Orjuela-Sanchez P, Ferreira MU: Plasmodium vivax: microsatellite analysis of multiple-clone infections. Exp Parasitol 2008, 120:330-336.

59. Karunaweera ND, Ferreira MU, Hart DL, Wirth DF: Fourteen polymorphic microsatellite DNA markers for the human malaria parasite Plasmodium vivax. Mol Ecol Notes 2007, 7:172-175.

60. Duan J, Mu J, Thera MA, Joy D, Kosakovsky Pond SL, Diemert D, Long C, Zhou H, Miura K, Ouattara A, Dolo A, Doumbo O, Su XZ, Miller L: Population structure of the genes encoding the polymorphic Plasmodium falciparum apical membrane antigen 1: implications for vaccine design. Proc Natl Acad Sci USA 2008, 105:7857-7862.

61. Wang X, Mu J, Li G, Chen P, Guo X, Fu L, Chen L, Su X, Wellems TE: Decreased prevalence of the Plasmodium falciparum chloroquine resistance transporter 76T marker associated with cessation of chloroquine use against $P$. falciparum malaria in Hainan, People's Republic of China. Am J Trop Med Hyg 2005, 72:410-414.

62. Cheng $Q$, Saul A: Sequence analysis of the apical membrane antigen I (AMA-1) of Plasmodium vivax. Mol Biochem Parasitol 1994, 65:183-187.

63. Gene Codes Corporation: Sequencher ${ }^{\circledR}$ version 5.0 sequence analysis software. In Ann Arbor, MI, USA; http://www.genecodes.com.

64. Ntumngia FB, McHenry AM, Barnwell JW, Cole-Tobian J, King CL, Adams JH: Genetic variation among Plasmodium vivax isolates adapted to non-human primates and the implication for vaccine development. Am J Trop Med Hyg 2009, 80:218-227.

65. Tamura K, Peterson D, Peterson N, Stecher G, Nei M, Kumar S: MEGA5: molecular evolutionary genetics analysis using maximum likelihood, 
evolutionary distance, and maximum parsimony methods. Mol Biol Evol 2011, 28:2731-2739.

66. Librado P, Rozas J: DnaSP v5: a software for comprehensive analysis of DNA polymorphism data. Bioinformatics 2009, 25:1451-1452.

67. Nei M, Gojobori T: Simple methods for estimating the numbers of synonymous and nonsynonymous nucleotide substitutions. Mol Biol Evol 1986, 3:418-426.

68. Hurlbert SH: The non-concept of species diversity: a critique and alternative parameters. Ecology 1971, 52:577-586.

69. Goudet J: FSTAT (Version 1.2): a computer program to calculate F-statistics $J$ Hered 1995, 86:485-486.

70. Glaubitz JC: CONVERT: a user friendly program to reformat diploid genotypic data for commonly used population genetic software packages. Mol Ecol Notes 2004, 4:309-310.

71. Akey JM, Eberle MA, Rieder MJ, Carlson CS, Shriver MD, Nickerson DA, Kruglyak L: Population history and natural selection shape patterns of genetic variation in 132 genes. PLoS Biol 2004, 2:e286.

72. Hudson RR: Estimating the recombination parameter of a finite population model without selection. Genet Res 1987, 50:245-250.

73. Lewontin RC: The interaction of selection and linkage. I. General considerations; heterotic models. Genetics 1964, 49:49-67.

74. Hill WG, Robertson A: Linkage disequilibrium in finite populations. Theor Appl Genet 1968, 38:226-231.

75. Bradbury PJ, Zhang Z, Kroon DE, Casstevens TM, Ramdoss Y, Buckler ES: TASSEL: software for association mapping of complex traits in diverse samples. Bioinformatics 2007, 23:2633-2635.

76. Mueller JC: Linkage disequilibrium for different scales and applications. Brief Bioinform 2004, 5:355-364.

77. Coley AM, Gupta A, Murphy VJ, Bai T, Kim H, Foley M, Anders RF, Batchelor AH: Structure of the malaria antigen AMA1 in complex with a growth-inhibitory antibody. PLOS Pathog 2007, 3:1308-1319.

78. Srinivasan P, Beatty WL, Diouf A, Herrera R, Ambroggio X, Moch JK, Tyler JS, Narum DL, Pierce SK, Boothroyd JC, Haynes JD, Miller LH: Binding of Plasmodium merozoite proteins RON2 and AMA1 triggers commitment to invasion. Proc Natl Acad Sci USA 2011, 108:13275-13280

79. Dutta S, Lee SY, Batchelor AH, Lanar DE: Structural basis of antigenic escape of a malaria vaccine candidate. Proc Natl Acad Sci USA 2007, 104:12488-12493.

80. Dutta S, Dlugosz LS, Drew DR, Ge X, Ababacar D, Rovira YI, Moch JK, Shi M, Long CA, Foley M, Beeson JG, Anders RF, Miura K, Haynes JD, Batchelor AH: Overcoming antigenic diversity by enhancing the immunogenicity of conserved epitopes on the malaria vaccine candidate apical membrane antigen-1. PLoS Pathog 2013, 9:e1003840.

81. Collins CR, Withers-Martinez C, Bentley GA, Batchelor AH, Thomas AW, Blackman MJ: Fine mapping of an epitope recognized by an invasion-inhibitory monoclonal antibody on the malaria vaccine candidate apical membrane antigen 1. J Biol Chem 2007, 282:7431-7441.

82. Kasehagen LJ, Mueller I, McNamara DT, Bockarie MJ, Kiniboro B, Rare L, Lorry K, Kastens W, Reeder JC, Kazura JW, Zimmerman PA: Changing patterns of Plasmodium blood-stage infections in the Wosera region of Papua New Guinea monitored by light microscopy and high throughput PCR diagnosis. Am J Trop Med Hyg 2006, 75:588-596.

83. Riley EM, Stewart VA: Immune mechanisms in malaria: new insights in vaccine development. Nat Med 2013, 19:168-178.

84. Richards JS, Beeson JG: The future for blood-stage vaccines against malaria. Immunol Cell Biol 2009, 87:377-390.

85. Ferreira MU, da Silva NM, Wunderlich G: Antigenic diversity and immune evasion by malaria parasites. Clin Diagn Lab Immunol 2004, 11:987-995.

86. Polley SD, Conway DJ: Strong diversifying selection on domains of the Plasmodium falciparum apical membrane antigen 1 gene. Genetics 2001, 158:1505-1512.

87. Lal AA, Hughes MA, Oliveira DA, Nelson C, Bloland PB, Oloo AJ, Hawley WE, Hightower AW, Nahlen BL, Udhayakumar V: Identification of T-cell determinants in natural immune responses to the Plasmodium falciparum apical membrane antigen (AMA-1) in an adult population exposed to malaria. Infect Immun 1996, 64:1054-1059.

88. Udhayakumar V, Kariuki S, Kolczack M, Girma M, Roberts JM, Oloo AJ, Nahlen BL, Lal AA: Longitudinal study of natural immune responses to the Plasmodium falciparum apical membrane antigen (AMA-1) in a holoendemic region of malaria in western Kenya: Asembo Bay Cohort Project VIII. Am J Trop Med Hyg 2001, 65:100-107.
89. Cui L, Escalante AA, Imwong M, Snounou G: The genetic diversity of Plasmodium vivax populations. Trends Parasitol 2003, 19:220-226.

90. Yung AP, Bennett NM: Chloroquine-resistant falciparum malaria in Papua New Guinea. Med J Aust 1976, 2:320-321.

91. Grimmond TR, Donovan KO, Riley ID: Chloroquine resistant malaria in Papua New Guinea. P N G Med J 1976, 19:184-185.

92. Rieckmann $\mathrm{KH}$, Davis DR, Hutton DC: Plasmodium vivax resistance to chloroquine? Lancet 1989, 2:1183-1184.

93. Whitby M, Wood G, Veenendaal JR, Rieckmann K: Chloroquine-resistant Plasmodium vivax. Lancet 1989, 2:1395.

94. Koepfli C, Ross A, Kiniboro B, Smith TA, Zimmerman PA, Siba P, Mueller Felger I: Multiplicity and diversity of Plasmodium vivax infections in a highly endemic region in Papua New Guinea. PLoS Negl Trop Dis 2011 5:e1424.

doi:10.1186/1475-2875-13-233

Cite this article as: Arnott et al:: Distinct patterns of diversity, population structure and evolution in the AMA1 genes of sympatric Plasmodium falciparum and Plasmodium vivax populations of Papua New Guinea from an area of similarly high transmission. Malaria Journal 2014 13:233.

\section{Submit your next manuscript to BioMed Central and take full advantage of:}

- Convenient online submission

- Thorough peer review

- No space constraints or color figure charges

- Immediate publication on acceptance

- Inclusion in PubMed, CAS, Scopus and Google Scholar

- Research which is freely available for redistribution 